\title{
IGR J17497-2821: a new X-ray nova
}

\author{
R. Walter ${ }^{1,2}$, P. Lubiński ${ }^{3,1}$, S. Paltani ${ }^{1,2}$, N. Produit ${ }^{1,2}$, J. Zurita $^{4}$, E. Kuulkers ${ }^{5}$, V. Beckmann ${ }^{6,7}$, N. Gehrels ${ }^{6}$, \\ A. Blecha ${ }^{2}$, F. Carrier ${ }^{8}$, and M. Cherix ${ }^{2}$ \\ 1 INTEGRAL Science Data Centre, Chemin d'Écogia 16, 1290 Versoix, Switzerland \\ 2 Geneva Observatory, University of Geneva, Chemin des Maillettes 51, 1290 Sauverny, Switzerland \\ e-mail: Roland.Walter@obs.unige.ch \\ 3 N. Copernicus Astronomical Center, Bartycka 18, 00-716 Warsaw, Poland \\ 4 AIM - Astrophysique Interactions Multi-Échelles, CEA Saclay, DSM/DAPNIA/SAp, Bât. 709, L'Orme des Merisiers, \\ 91191 Gif-sur-Yvette Cedex, France \\ 5 ISOC, ESAC/ESA, Apartado 50727, 28080 Madrid, Spain \\ 6 NASA Goddard Space Flight Center, Astrophysics Science Division, Greenbelt, MD 20771, USA \\ 7 University of Maryland Baltimore County, Joint Center for Astrophysics, 1000 Hilltop Circle, Baltimore, MD 21250, USA \\ 8 Instituut voor Sterrenkunde, Katholieke Universiteit Leuven, Celestijnenlaan 200D, 3001 Leuven, Belgium
}

Received 7 October 2006 / Accepted 17 November 2006

\section{ABSTRACT}

\begin{abstract}
Context. A new hard X-ray transient has been discovered by INTEGRAL on 2006 Sep. 17 at 1 degree from the Galactic Centre. Aims. INTEGRAL, Swift/XRT and optical photometric observations are used to characterize IGR J17497-2821 and to unveil its nature. Methods. The X-ray position has been refined to arcsec level through a study of the optical variability of the possible counterparts. Hard X-ray variability and spectroscopy are used to determine the nature of the source outburst.

Results. IGR J17497-2821 is a new X-ray Nova in low-hard state, probably a new Black-Hole Candidate.
\end{abstract}

Key words. gamma rays: observations - X-rays: individuals: IGR J17497-2821

\section{Introduction}

The large field of view and good spatial resolution of the Imager on board the International Gamma-Ray Astrophysics Laboratory (INTEGRAL; Winkler et al. 2003) allows to monitor regularly a large fraction of the Galactic bulge. The detection of new X-ray Novae has always been among the important scientific goals of the mission as 3 out of 4 contain Black Hole Candidates (Casares 2004).

A new hard X-ray source, IGR J17497-2821, has been discovered by INTEGRAL on September 17, 2006 (Soldi et al. 2006) during the real-time routine monitoring performed at the INTEGRAL Science Data Centre (Courvoisier et al. 2003).

The source, located at a distance of $1.06^{\circ}$ from the Galactic center, was first detected at a level of $25 \mathrm{mCrab}$ in a sky image obtained for spacecraft pointing 04790063 by IBIS/ISGRI (Ubertini et al. 2003; Lebrun et al. 2003). The source reappeared $4 \mathrm{~h}$ later and its flux then increased up to a level of $f_{20-200 \mathrm{kev}}=2.6 \times 10^{-9} \mathrm{erg} \mathrm{s}^{-1} \mathrm{~cm}^{-2}(0.13 \mathrm{Crab})$ in $400 \mathrm{ks}$.

In this letter we present INTEGRAL data obtained on the source from the first detection up to October 5, 2006 using observing time obtained through the AO-4 Key Program (Winkler 2006) and through the Galactic bulge monitoring program (Kuulkers et al. 2006). We also present results obtained from two Swift/XRT (Gehrels et al. 2004) observations carried out on September 19 and 22. In addition we discuss results of photometric observations obtained on September 22 with the "Euler" telescope from La Silla.

The observations and data analysis are presented in Sect. 2. The source position and possible optical/infrared counterparts are discussed in Sect. 3. The hard X-ray light curve obtained with INTEGRAL is presented and analyzed in Sect. 4 . The broadband source spectrum and its variability are presented in Sect. 5. Finally, Sect. 6 provides a discussion on the nature of this new transient.

\section{Hard X-ray observations}

\subsection{INTEGRAL observations}

In the frame of this letter we analyzed 393 INTEGRAL spacecraft pointings of the Galactic bulge field available from spacecraft revolution 479 to 485 (one spacecraft revolution spans about 3 days) i.e. from 2006 Sep. 15 02:35 to 2006 Oct. 5 13:22 (UTC). While IGR J17497-2821 is located within the IBIS/ISGRI field of view for most of the pointings, it was within the JEM-X1 (Lund et al. 2003) field of view for only 81 pointings. The data were analyzed using the INTEGRAL Offline Scientific Analysis (OSA) software ${ }^{1}$. We used OSA version 6.0 for most analysis except for the spectral analysis that was carried with OSA 5.1 (for lack of final OSA 6.0 spectral responses). The ISGRI analysis was performed with an input catalogue of 55 bright sources, which have been detected in a mosaic image of the field. For spectral analysis the data set was split in 3 periods corresponding to the onset, maximum and decay part of the outburst.

We also used public INTEGRAL data obtained before the outburst in order to derive an upper limit to the source flux in quiescence and to search for type 1 burst.

\footnotetext{
${ }^{1}$ Available from the ISDC website: http://isdc. unige.ch/
} 


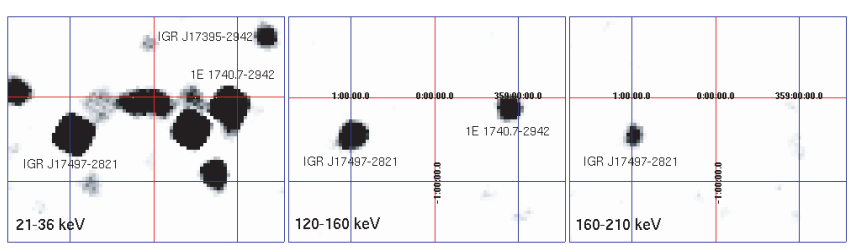

Fig. 1. INTEGRAL IBIS/ISGRI images of the field in three energy bands (galactic coordinates). IGR J17497-2821 outshines the "great annihilator" $1 \mathrm{E}$ 1740.7-2942 in all bands.

Table 1. List of Swift/XRT observations.

\begin{tabular}{lllr}
\hline \hline ID & OBS ID & Time [UTC] & Eff. exposure \\
\hline S1 & 00030805001 & 2006 Sep. 19 15:51-16:17 & $1432 \mathrm{~s}$ \\
S2 & 00230504991 & 2006 Sep. 22 06:46-08:22 & 489 s \\
\hline
\end{tabular}

\subsection{Swift/XRT observations}

Two Swift/XRT observations of IGR J17497-2821 were analyzed. The observations are listed in Table 1. Both observations were performed in XRT photon counting mode. In both observations the source location on the detector plane coincides with dead detector columns, due to micrometeorite impact. The analysis has been performed using HEAsoft version 6.1.12. Source counts were extracted within 2 arcmin radius around the source position. The dead detector columns have been accounted for by correcting the exposure map, resulting in a 50\% decrease of the area response function (ARF). Response functions (ARF \& $\mathrm{RMF}$ ) used in the analysis are based on the input provided by the instrument team in April 2006. The systematic error of the response matrix is about $3 \%$, and the total flux uncertainty in the $2-10 \mathrm{keV}$ band is about $10 \%$. The individual Swift/XRT observations do not show any significant source flux variation.

\section{Source position and optical counterpart}

The INTEGRAL IBIS/ISGRI mosaic sky images, created in 3 energy bands, are given in Fig. 1. IGR J17497-2821 is the brightest source in the Galactic center region above $100 \mathrm{keV}$. Note also the presence of a close-by second new transient source, IGR J17395-2942, at RA $=17^{\mathrm{h}} 39.5^{\prime}$, Dec $=-29^{\circ} 42^{\prime}(\mathrm{J} 2000$, with a $3 \sigma$ uncertainty of 1.2 arcmin).

We have determined the position of IGR J17497-2821 using a 21-161 keV mosaic image of the field built using data from revolution 480 and 481 when the source was in its brightest state and its significance reached $245 \sigma$. The resulting position is RA $=17^{\mathrm{h}} 49^{\prime} 38.0^{\prime \prime}$ Dec $=-28^{\circ} 21^{\prime} 14^{\prime \prime}(\mathrm{J} 2000)$ with a $3 \sigma$ statistical uncertainty of 2.4 arcsec (a systematic uncertainty of 14 arcsec should be added to take into account uncertainties on the misalignment).

We used the Swift/XRT image derived from the first observation to obtain a source position of RA $=17^{\mathrm{h}} 49^{\prime} 38.0^{\prime \prime}$ Dec $=-28^{\circ} 21^{\prime} 18^{\prime \prime}(\mathrm{J} 2000,5$ arcsec uncertainty $)$ which includes a catalogued blended near infrared source (2MASS J17493780-2821181) (Walter et al. 2006).

Laycock et al. (2006) used the Swift/XRT position to report a list of possible counterparts observed in a series of $C T I O / M O S A I C$ frames obtained in 2003 in various optical and infrared bands. Following the discovery of the source we obtained an image in the I-band with the C2 CCD-Camera

\footnotetext{
2 Available from the GSFC website:

http://swift.gsfc.nasa.gov/
}

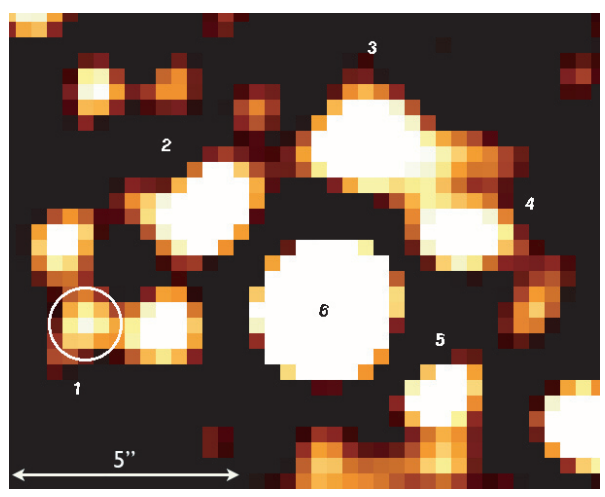

Fig. 2. Stacked and deconvolved CCD images obtained in the $I$ band with the C2 camera mounted on the "Euler" telescope (North is up; $0.34 \mathrm{arcsec} / \mathrm{pixel})$. The numbers identify the counterparts that have been studied and for which 2003 photometry was provided by Laycock et al. (2006). The circle indicates the probable position of IGR J17497-2821, based on the photometric results.

Table 2. I magnitudes obtained for a sample of faint stars on 2006 Sep. 22 01:30 (UTC) and in archived CTIO observations of 2003. The uncertainties on the $\mathrm{C} 2$ magnitudes include systematic errors related to the photometric calibration. The stellar positions and CTIO magnitudes are from Laycock et al. (2006). The identifications correspond to Fig. 2.

\begin{tabular}{ccccc}
\hline \hline ID & RA & Dec & $I_{\text {C2 } 2006}$ & $I_{\text {CTIO } 2003}$ \\
& J2000 & J 2000 & Mag. & Mag. \\
\hline 1 & $17^{\mathrm{h}} 49^{\prime} 38.11^{\prime \prime}$ & $-28^{\circ} 21^{\prime} 17.2^{\prime \prime}$ & $20.54 \pm 0.17$ & $21.31 \pm 0.09$ \\
2 & $17^{\mathrm{h}} 49^{\prime} 37.92^{\prime \prime}$ & $-28^{\circ} 21^{\prime} 14.8^{\prime \prime}$ & $19.65 \pm 0.09$ & $19.78 \pm 0.03$ \\
3 & $17^{\mathrm{h}} 49^{\prime} 37.67^{\prime \prime}$ & $-28^{\circ} 21^{\prime} 13.4^{\prime \prime}$ & $19.58 \pm 0.08$ & $19.69 \pm 0.02$ \\
4 & $17^{\mathrm{h}} 49^{\prime} 37.51^{\prime \prime}$ & $-28^{\circ} 21^{\prime} 15.6^{\prime \prime}$ & $20.17 \pm 0.12$ & $20.07 \pm 0.05$ \\
5 & $17^{\mathrm{h}} 49^{\prime} 37.55^{\prime \prime}$ & $-28^{\circ} 21^{\prime} 19.0^{\prime \prime}$ & $20.29 \pm 0.14$ & $20.16 \pm 0.05$ \\
6 & $17^{\mathrm{h}} 49^{\prime} 37.72^{\prime \prime}$ & $-28^{\circ} 21^{\prime} 16.9^{\prime \prime}$ & $15.14 \pm 0.02$ & $15.14 \pm 0.01$ \\
\hline
\end{tabular}

(Davignon et al. 2004) mounted on the "Euler" $1.2 \mathrm{~m}$ telescope at La Silla on 2006 Sep. 22 00:00-03:00 (UTC). 50 frames were obtained, cleaned, stacked and deconvolved (Fig. 2). Aperture photometry was performed on the deconvolved image using IRAF/DAOPHOT ${ }^{3}$ removing each time the contribution of close-by sources by fitting their point spread function. The aperture radius was set to 4 pixels ( $1.36 \mathrm{arcsec})$ to allow for the detection of variability in blended sources (the seeing $F W H M$ varied between 1.3 and 3 arcsec during the observation). Photometric calibration has been obtained by comparing our results with the magnitude of Laycock et al. (2006). The resulting magnitudes for 6 counterpart candidates are listed in Table 2 and compared to the magnitude of 2003.

The 2006 I magnitude of 5 counterpart candidates match very well with the 2003 values indicating no variability. However, the apparent flux of candidate 1 with position RA = $17^{\mathrm{h}} 49^{\prime} 38.11^{\prime \prime}$, Dec $=-28^{\circ} 21^{\prime} 17.2^{\prime \prime}$ (Laycock et al. 2006) increased significantly by $\Delta I=0.77 \pm 0.19 \mathrm{mag}$ (a $4 \sigma$ detection) which tentatively identifies the X-ray transient counterpart position. This position is within 1 arcsec of the X-ray source position recently obtained by Chandra (Paizis et al. 2006).

The optical and infrared photometry (Laycock et al. 2006) of candidate 1 measured in 2003 indicate that it can be a foreground low mass star or a massive star located at the distance of the galactic center. An undetected solar type star at the center of our Galaxy $\left(11^{\mathrm{mag}}<A_{V}<23^{\mathrm{mag}}\right)$, blended with candidate 1 ,

\footnotetext{
3 Available from the NOAO website: http://iraf.noao.edu/
} 


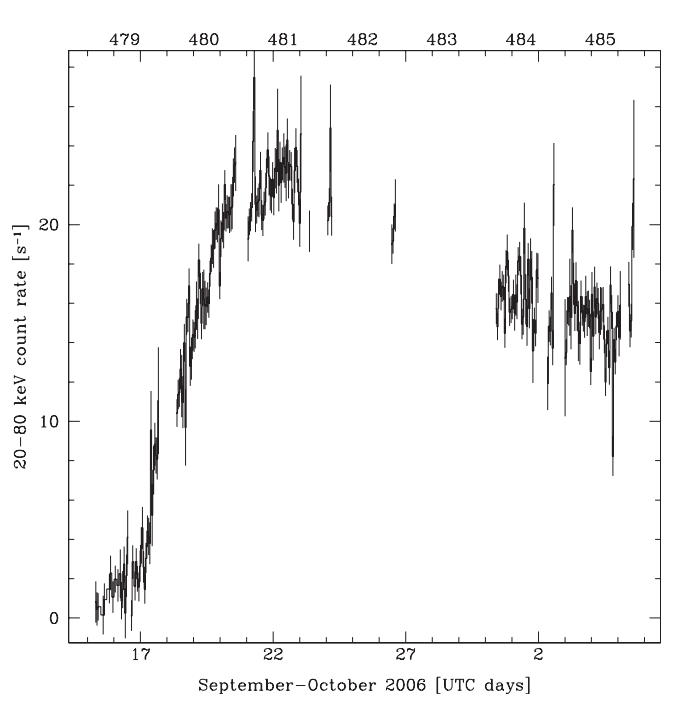

Fig. 3. IBIS/ISGRI (20-80 keV) lightcurve of IGR J17497-2821, rebinned in bins of $4000 \mathrm{~s}$. The numbers given at the top indicate the INTEGRAL spacecraft revolutions.

would have $24^{\mathrm{mag}}<I<30^{\mathrm{mag}}$ compatible with the photometric information of 2003. The measured variation of the $I$ magnitude is likely related to such a blended star and the magnitude of the $\mathrm{X}$-ray transient in outburst can be estimated as $I \sim 21.5$ suggesting a flux increase by a factor of 25 to 2500 .

\section{Hard X-ray lightcurve}

We constructed the hard X-ray lightcurve of IGR J17497-2821, in the 20 to $80 \mathrm{keV}$ spectral band, using the INTEGRAL IBIS/ISGRI data. The lightcurve (Fig. 3) spans 20 days with a time bin of $100 \mathrm{~s}$. The source outburst started on $2006 \mathrm{Sep} .16$ 23:48 (UTC) with one short flare and developed in a fast-rise exponential-decay (FRED) outburst typical of X-ray Novae. The source flux rose in $400 \mathrm{ks}$ from less than $f_{20-200 \mathrm{keV}}=3 \times$ $10^{-10} \mathrm{erg} \mathrm{s}^{-1} \mathrm{~cm}^{-2}$ to a maximum of $2.6 \times 10^{-9} \mathrm{erg} \mathrm{s}^{-1} \mathrm{~cm}^{-2}$. The maximum was reached at the beginning of revolution 481 ( $\approx 2006$, Sep. 21.5 UTC) for energies above $50 \mathrm{keV}$, and at the end of revolution $481(\approx 2006$, Sep. 22.9 UTC) at lower energies (20-40 keV). Since then the source flux is decreasing exponentially with a time constant of 2 weeks.

The hard X-ray lightcurve features flickering on short time scales. Using a time binning of $1 \mathrm{ks}$, the variability $\mathrm{rms}$ is twice larger than expected from the measurement uncertainties. The amplitude of this flickering reaches $10 \%$. The detailed study of these variations is out of the scope of this letter.

A mosaic of all public INTEGRAL IBIS/ISGRI pointing images obtained from revolution 30 to revolution 301 (2005-03-31 17:53) was analysed to estimate an upper limit of the source quiescent flux. No excess was detected at the position of the source and a $5 \sigma$ upper limit for the flux in quiescence can be estimated at 0.052 counts $\mathrm{s}^{-1}$ in the $26-51 \mathrm{keV}$ energy range (i.e. $0.6 \mathrm{mCrab}$ ), for an effective exposure time of $1.5 \mathrm{Ms}$. This upper limit and effective exposure includes systematic errors related to the cleaning of the image from bright sources.

We also searched the INTEGRAL archive for signature of type 1 burst by producing a JEM-X 3-10 keV light-curve with a time bin of $30 \mathrm{~s}$. We kept all time bins with effective exposure time above $25 \mathrm{~s}$. Over a total of $995 \mathrm{ks}$ we did not found any short time scale burst at a limiting sensitivity of about

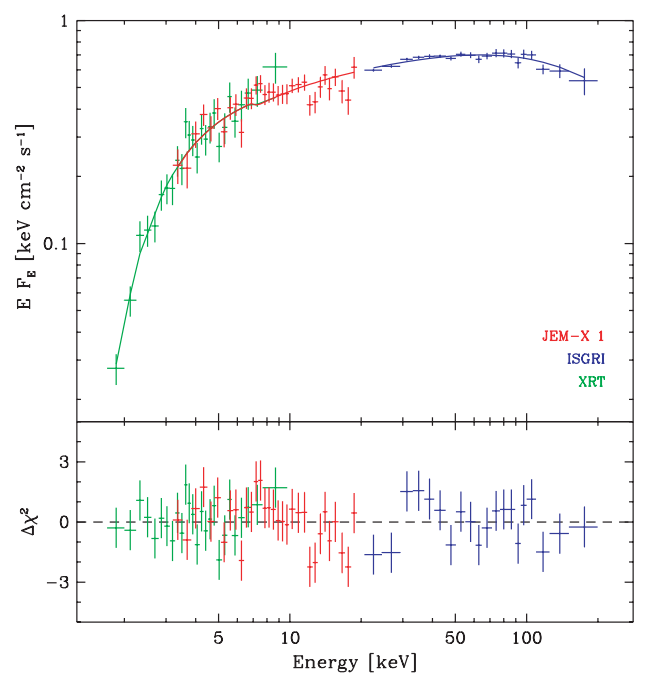

Fig. 4. Best fit cut-off power-law model for the Swift/XRT S2 and INTEGRAL/JEM-X1 and IBIS/ISGRI spectra of revolution 481. The normalizations have been adjusted.

Table 3. Results of spectral fitting close to outburst maximum. We used INTEGRAL (ISGRI\&JEM-X1) data from revolution 481 and either the first or the second Swift/XRT observation. Two models have been tried: either a simple absorbed power-law or an absorbed cut-off power-law.

\begin{tabular}{lccccc}
\hline \hline XRT & $\chi_{v}^{2}$ & $\begin{array}{c}N_{\mathrm{H}} \\
10^{22} \mathrm{~cm}^{-2}\end{array}$ & $\Gamma$ & $\begin{array}{c}I_{1 \mathrm{keV}} \\
\mathrm{ph} / \mathrm{s} \mathrm{cm}^{2} \mathrm{keV}\end{array}$ & $\begin{array}{c}E_{\text {cut }} \\
\mathrm{keV}\end{array}$ \\
\hline \multicolumn{5}{l}{ Power-law: } \\
S1 & $1.75_{96}$ & $5.1 \pm 0.3$ & $1.93 \pm 0.02$ & $0.51 \pm 0.04$ & - \\
S2 & $1.87_{73}$ & $5.5 \pm 0.5$ & $1.93 \pm 0.02$ & $0.51 \pm 0.04$ & - \\
\multicolumn{2}{l}{ Cut-off power-law: } & & & \\
S1 & $1.22_{95}$ & $4.4 \pm 0.3$ & $1.67 \pm 0.06$ & $0.252 \pm 0.005$ & $197_{-34}^{+70}$ \\
S2 & $1.14_{72}$ & $4.5 \pm 0.4$ & $1.67 \pm 0.06$ & $0.248 \pm 0.004$ & $195_{-37}^{58}$ \\
\hline
\end{tabular}

$L_{\mathrm{bol}} \approx(0.1-0.2) \times L_{\mathrm{EDD}}$ for a source at the distance of the galactic centre.

\section{Hard X-ray spectroscopy}

The INTEGRAL/JEM-X1 and IBIS/ISGRI average spectra of IGR J17497-2821 obtained in revolution 481 were used together with the Swift/XRT spectrum obtained from one or the other of the two observations. The combined spectra were fit with either an absorbed power-law or an absorbed cut-off power-law model. Table 3 gives the resulting best fit model parameters obtained with XSPEC version 11 (Arnaud 1996).

While the simple power-law model is not adequate, the data are well represented by a cut-off power-law (Fig. 4). The spectral cut-off is detected with a high significance. The average spectrum is very hard with a power-law index of $\Gamma=1.67 \pm 0.06$. The continuum can also be represented by a comptonization model (comptt), in which case a best fit optical depth of $\tau=1.45_{-1.4}^{+0.5}$ and electronic temperature of $k T=35_{-9}^{+200} \mathrm{keV}$ are obtained. Because both parameters are strongly correlated, they are not well constrained by the fit though. The best fit absorbing column density (Table 3 ) is significantly higher than the interstellar column density derived from $21 \mathrm{~cm}$ data $\left(1.3 \times 10^{22} \mathrm{~cm}^{-2}\right)$.

Cross calibration factors were used in the model fitting to account for cross calibration and for source variability. The spectral cut-off can be detected using ISGRI data alone therefore it is 


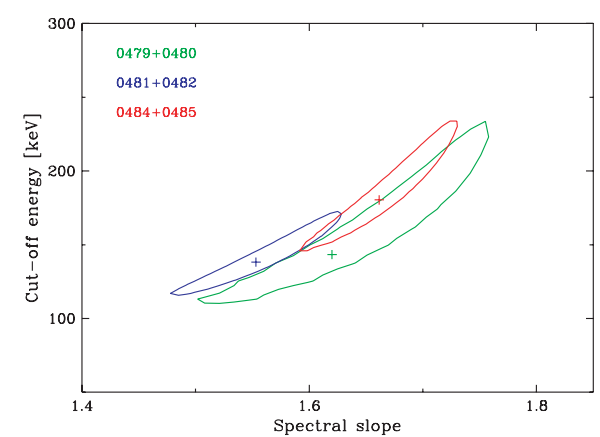

Fig. 5. Probability contours $\left(\Delta \chi^{2}=2.3\right)$ for the power-law slope and cut-off energy for three epochs. This is based on ISGRI data alone.

not due to these factors. Accurate spectral modeling will require additional effort that is out of the scope of the present letter.

We applied the absorbed cut-off power-law model to the INTEGRAL ISGRI average spectra built for the onset (revolution 479-480), maximum (revolution 481-482) and decay (revolution 484-485) part of the outburst and derived confidence contours for the power-law slopes and cut-off energies for the three data sets (Fig. 5). In the absence of X-ray spectra the uncertainties are such that spectral variations cannot be very well constrained at this stage of the analysis. There is a tendency however for the cut-off energy and the spectral slope to decrease when the source flux increases.

\section{Discussion}

The fast-rise exponential-decay lightcurve of the outburst of IGR J17497-2821, the time scale involved, the observed variation at high energy by a factor $>400$ from quiescence and the increase of the optical flux by 3 to 9 mag are canonical signatures of an X-ray Nova (e.g. Chen et al. 1997).

Observations of X-ray Novae have shown that their high energy spectra can change from a soft to a hard state when the $\mathrm{X}$-ray luminosity decreases below about $10^{37} \mathrm{erg} \mathrm{s}^{-1}$ (Tanaka \& Shibazaki 1996). The soft state is characterized by thermal emission with a typical temperature of $\sim 1 \mathrm{keV}$ and by a steep hard tail power law $(\Gamma \sim 2-3)$. The low-hard state is instead characterized by a spectrum dominated by non thermal emission $(\Gamma \sim 1.5-2)$ (Grove et al. 1998) and featuring a high energy spectral cut-off.

Typical X-ray Nova models use the inner parts of a thin accretion disc to explain the soft X-ray thermal spectral component and inverse Compton emission by some form of hot plasma to account for the hard X-ray emission. If the mass accretion rate does not exceed the rate at which the disk evaporates in the corona, the disk may not extend to the last stable orbit and the spectrum does not reach the soft state. The corresponding limiting accretion luminosity is $10^{37.2} \mathrm{erg} \mathrm{s}^{-1}$ (Meyer et al. 2000).

The peak hard X-ray flux reached during the outburst of IGR J17497-2821 is $2.3 \times 10^{-9} \mathrm{erg} \mathrm{s}^{-1} \mathrm{~cm}^{-2}(20-200 \mathrm{keV})$ which corresponds to a luminosity $\geq 1.7 \times 10^{37} \mathrm{erg} \mathrm{s}^{-1}$ (20-200 keV), assuming that IGR J17497-2821 is located close or behind the Galactic center region (which is supported by the large observed absorbing column density).

The outburst peak luminosity is therefore in agreement with the absence of a soft spectral component and one can predict that
IGR J17497-2821 will stay in the hard state unless it brightens again. The current outburst of IGR J17497-2821 very likely belongs to the specific category of X-ray Novae that never reaches the high soft state and remains dominated by the hard X-ray emission for the complete outburst (Brocksopp et al. 2004).

In the low-hard spectral state, source flickering at a level of $10 \%$ and correlation between source flux and hard X-ray spectral shape have been observed in several X-ray Novae (Kalemci et al. 2004; Yamaoka et al. 2005). Similar behavior seems to be observed in IGR J17497-2821.

In neutron star systems, type I bursts are expected on average every $27 \mathrm{ks}$ and most of them with a luminosity of $0.1 \times L_{\mathrm{EDD}}$ (Remillard et al. 2006). The absence of any bright type I burst in IGR J17497-2821 over $995 \mathrm{ks}$, together with a cut-off energy of $200 \mathrm{keV}$, a Compton optical depth $\leq 2$ and a hard X-ray luminosity above $10^{37} \mathrm{erg} / \mathrm{s}$ strongly suggest that IGR J17497-2821 harbours a black-hole candidate (Barret et al. 2000).

Follow-up observations of IGR J17497-2821 will allow further studies of the source.

Acknowledgements. Based on observations with INTEGRAL, an ESA project with instruments and science data centre funded by ESA member states (especially the PI countries: Denmark, France, Germany, Italy, Switzerland, Spain), Czech Republic and Poland, and with the participation of Russia and the USA. We would like to thank J. Hill for assistance in analysing the Swift/XRT data and D. Ségransan for performing the observations at the "Euler" telescope. P.L. was supported by KBN grant 1P03D01128.

\section{References}

Arnaud, K. A. 1996, in Astronomical Data Analysis Software and Systems V, ed. G. H. Jacoby, \& J. Barnes, ASP Conf. Ser., 101, 17

Barret, D., Olive, J. F., Boirin, L., et al. 2000, ApJ, 533, 329

Brocksopp, C., Bandyopadhyay, R. M., \& Fender, R. P. 2004, New Astron., 9, 249

Casares, J. 2004, in The Many Scales of the Universe - JENAM 2004 Astrophys. Rev., ed. J. C. Del Toro Iniesta

Chen, W., Shrader, C. R., \& Livio, M. 1997, ApJ, 491, 312

Courvoisier, T. J.-L., Walter, R., Beckmann, V., et al. 2003, A\&A, 411, L53

Davignon, G., Blecha, A., Burki, G., et al. 2004, in Ground-based Instrumentation for Astronomy, ed. A. F. M. Moorwood, \& I. Masanori, Proc. SPIE, 5492, 871

Gehrels, N., Chincarini, G., Giommi, P., et al. 2004, ApJ, 611, 1005

Grove, J. E., Johnson, W. N., Kroeger, R. A., et al. 1998, ApJ, 500, 899

Kalemci, E., Tomsick, J. A., Rothschild, R. E., Pottschmidt, K., \& Kaaret, P. 2004, ApJ, 603, 231

Kuulkers, E., Shaw, S. E., Brandt, S., et al. 2006, in The Transient Milky Way: A Perspective for MIRAX, ed. J. Braga, F. D’Amico, \& R. E. Rothschild, AIP Conf. Proc., 840, 30

Laycock, S., Zhao, P., Berg, M. V. D., Grindlay, J., \& Hong., J. 2006, The Astronomer's Telegram, 895, 1

Lebrun, F., Leray, J. P., Lavocat, P., et al. 2003, A\&A, 411, L141

Lund, N., Budtz-Jørgensen, C., Westergaard, N. J., et al. 2003, A\&A, 411, L231

Meyer, F., Liu, B. F., \& Meyer-Hofmeister, E. 2000, A\&A, 354, L67

Paizis, A., Nowak, M., Rodriguez, J., et al. 2006, The Astronomer's Telegram, 907, 1

Remillard, R. A., Lin, D., Cooper, R. L., \& Narayan, R. 2006, ApJ, 646, 407

Soldi, S., Walter, R., Eckert, D., et al. 2006, The Astronomer's Telegram, 885, 1

Tanaka, Y., \& Shibazaki, N. 1996, ARA\&A, 34, 607

Ubertini, P., Lebrun, F., Di Cocco, G., et al. 2003, A\&A, 411, L131

Walter, R., Eckert, D., Kreykenbohm, I., et al. 2006, The Astronomer's Telegram, 889,1

Winkler, C. 2006, AO-4 Key Programme, INTEGRAL Science Operation Centre, ESA

Winkler, C., Courvoisier, T. J.-L., Di Cocco, G., et al. 2003, A\&A, 411, L1

Yamaoka, K., Uzawa, M., Arai, M., Yamazaki, T., \& Yoshida, A. 2005, ChJA\&A, 5, 273 